Copyright (@ 2009 Institute of Electrical and electronics Engineers, Inc.

All Rights reserved.

Personal use of this material, including one hard copy reproduction, is permitted.

Permission to reprint, republish and/or distribute this material in whole or in part for any other purposes must be obtained from the IEEE.

For information on obtaining permission, send an e-mail message to stds-igr@ieee.org.

By choosing to view this document, you agree to all provisions of the copyright laws protecting it.

Individual documents posted on this site may carry slightly different copyright restrictions.

For specific document information, check the copyright notice at the beginning of each document. 


\title{
Applying Fractal and Chaos Theory to Animation in the Chinese Literati Tradition
}

\author{
Qing Huang, R.J. Balsys, \\ Centre for Intelligent and Networked Systems, \\ Central Queensland University, \\ Rockhampton, Qld., 4702. Australia. \\ q.huang@cqu.edu.au,balsys@cqu.edu.au
}

\begin{abstract}
This paper describes and tests the hypothesis that that which ancient Chinese philosophy searched for, the Tao, is the same principle as that found in modern Chaos and Fractal Theories. An elucidation of several key principles and notions of Chinese art theory is offered, coupled with fractal notions from Chaos theory. It is argued that Chinese art is an abstract form of symbolic brush-strokes, which elicit intrinsic mathematic values. An example of a modern evolving (animated) fractal form based on this notion is given to test our hypothesis.
\end{abstract}

Keywords-Tao, Chinese literati art, chaos, fractals, fractal dimension

\section{Introduction}

Chinese traditional art developed from a profoundly philosophical perspective. It is thoroughly a different approach to aesthetics to that of the West. Instead of being logically apprehensible as was the case in 19th Century Western Art culture, Chinese art attempts to capture the underlying truths and forces of Nature. Traditional Chinese art served this purpose. The Tao served as an underpinning for the philosophical basis of Chinese art from very early times.

Literally translated, Tao means "Way", "Tao cannot be conveyed by either words or silence. In that state, which is neither speech nor silence, its transcendental nature may be apprehended." (Cited in Sze 1959 [17].)

In attempting to bridge this unforeseeable gap, traditional Chinese art incorporates symbolic representation as a mean by which to elucidate universal, Taoist principles.

Tao then, is the universal underlying principle that informs and inheres in everything. It is the metaphysical framework and foundation of traditional Chinese philosophy and Art. Rather than attempting to verbalize what Tao is, Taoist sages would often try and attempt to represent Tao through esoteric charts and diagrams, that is to say, through visual symbols. This preference has often been identified as the origin of Chinese pictographic characters, Chinese calligraphy and Chinese paintings. The legacies of these aspects of Taoism include the emblems for Tao themselves, such as Tai-Ji (the Ying-Yang symbol), $B a$ $G u$, (the Eight trigrams), see Figure 1, and the sixty-four hexagrams.

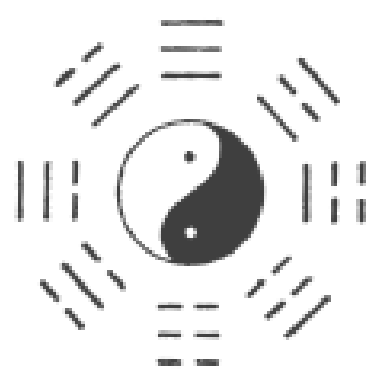

Figure 1: The Tai-Ji (Ying-yang) symbol and the eight trigrams.

In attempting to overcome the perceived language problems in explaining Tao, we will be utilizing concepts derived from modern-day notions in Fractals and Chaos theory, which we show are akin to Taoist metaphysical notions. Chaos theory similarly reveals some of the most fundamental and universal principles that constitute our universe.

"Physicists, biologists, astronomers and economists have created a new way of understanding the growth of complexity in nature. This new science, called chaos, offers a way of seeing order and pattern where formerly only the random, erratic, the unpredictable ... in short, the chaotic ... had been observed." (Gleick 1987 [6].)

In the sections that follow we examine the links between the Tao and modern fractal and chaos theory. We utilise this notion of fractals to develop animations that adhere to traditional fractal values found in Chinese art. 


\section{Fractals, Chaos Theory, the Tao and Chi- nese Artforms}

Chaos theory is quite an extensive field of research, incorporating a large range of epistemological knowledge, crossing many boundaries in a variety of scientific fields. This is similarly the case for Taoism, which also attempts to give a universal theory of everything in nature. Scientists of chaos theory, like their Taoist counterparts, also prefer to use charts, diagrams and computer-aided graphics in order to elucidate upon the intriguing concepts and notions of their theories. These graphics range from the so-called 'mathematical monsters' (The Cantor dust, The Sierpinski Gasket and The Koch Curves), to modern diagrams, such as the 'Lorenze attractor', the 'Barnsley's fern', and, most popular of all, the 'Mandelbrot set' (a very weird cardioid with infinite details of sprouts and tendrils along its boundary, like the tails of sea horses), see for instance the books by Barnseley[1] and Mandelbrot[11].

The purpose of these diagrams is similar to the Tai$J i$ and $B a-G u a$ diagrams in Taoism. These diagrams attempt to capture and visualize the hidden patterns of the unpredictable and chaotic behavior of nature in their images. For the Sages of Taoism, natural events seemed governed by pure chaos. In order to make sense of this seemingly incomprehensible world, traditional Chinese Taoist art served as a type of ordering mechanism, giving meaning to the world. Similarly, for modern-day scientists, armed with chaos theory, such diagrams go some way to explaining and interpreting the erratic and random sides of nature, which previously, could not be incorporated within a traditional scientific framework. In comparing several diagrams from these two seemingly disparate theories - coming as they do from worlds apart - a strong similarity emerges between them.

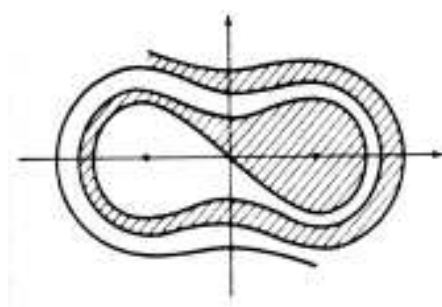

(a)

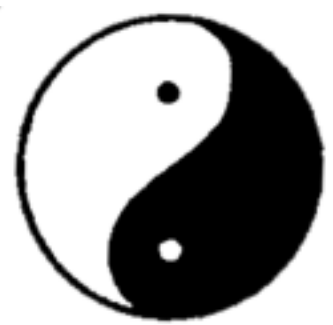

(b)
Figure 2: Similarity between the fractal basin boundary in the complex plane and the Ying-yang symbol. (a) The fractal basin boundary in the complex plane, (b) the Ying-yang symbol.

For example compare the figures in Figure 2. Figure 2(a) represents a so-called fractal basin boundary in a complex plane (phase image). It represents a dynamic sys- tem's long-term behavior, which in this instance has two equilibrium states. "Each equilibrium is an attractor and the boundary between two attractors can be complicated, which means chaos may appear at the boundary between one kind of steady behavior and another. (Gleick 1987 [6]). Figure 2(b) is the tai-ji diagram. Could this be an archaic prototype of a phase-space diagram, akin to the figure on the left? It represents a particular type of dynamic system behavior that ancient Taoists knew about. Could the spots in each zenith equilibrium state (in Chinese terminology, 'yin' and 'yang') be synchronistic with Figure 2 (a), which means attractors? The figure on the left represents a simple example of transforming algorithms and equations into form and shape. This is to help scientists and mathematicians visualize correlations within modern, chaos-theory geometry.

Zhang Yanyuan (an art critic of the Tang Dynasty) notes in his collection of essays on painting (Li Dai Ming Hua Ji), "By the Yin and Yang, innumerable forms are fashioned and produced, order is brought into chaos by their mysterious influence, while the indescribable spirit alone is revolving." (Sze 1959 [17].) Similarly, mathematicians and scientists made the same discovery when they note the infinite detailed richness of forms and structures revealing and unfolding along the boundary of Mandelbrot sets upon successive magnification. These mathematicians and scientists assume that various encyclopedic forms and shapes in nature might also be generated in an equivalent manner. Just like Zhuang Zi said: "That which causes things to be things is not itself a thing. The limit of each thing is called its boundary. The unlimited lies within the limited and though recognized within the limited, it is, nevertheless, unlimited." (Smith 1980 [16].)

The expanded experiment of complex plane images shows another parallel with Taoism. In metaphorical terms, mathematicians speak of the 'equipotential surface' of a certain subset, i.e. Mandelbrot set, or Julia set, as the force field. These are defined as surfaces on which the potential is constant. When scientists want to draw field lines (which represent the magnitude of the iterates) they use a method called binary decomposition, see Figure 3(a), which allows them to approximate arbitrary field lines of potential.

The derivation of the sixty-four hexagrams from the Tai$J i$ is astonishingly similar to this kind of method. As shown in Figure 3(b), the white bars indicate yang components while the dark bars indicate yin components. Each yin and yang component divides to give yet another pair of yin and yang components. Thus, the yin and yang in Tai-Ji (the innermost ring) divide to give the four directions (the second ring from center). Moving outwards, the next ring is formed to give eight directions. This is continued on until 
sixty-four hexagrams are achieved. See Wong 1997 [19].

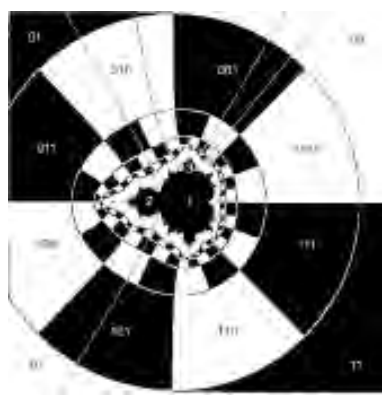

(a)

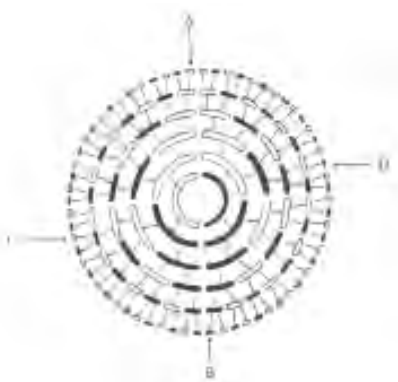

(b)
Figure 3: (a) Binary decomposition of the Julai set, (from Peitgen, 1992 [13]. (b) The 64 hexagrams.

The methodology employed by Chinese philosophers is termed by modern scientists as the addressing method. This system uses symbols or characters instead of numbers. When drawing field lines in the larger neighborhood of a Mandelbrot set, using the decomposition method, we find that there are also sixty-four cells. Is this merely a coincidence? We can see why Taoists used Tai-Ji and hexagrams to represent and explain invisible force fields. With the aid of modern day chaos theory and, in particular, the use of Mandelbrot sets, scientists do the same thing to explain such phenomena within these complex plane sets. These are the analogues of both Macro and Micro worlds, which are not directly apprehensible to the human senses; take the Earth's magnetic field for example. No surprise that Chinese sages invented such instruments as the compass to visualize and capture these invisible forces in the physical world.

Another example that exhibits the similarity between Taoism and Chaos theory is a mathematical diagrammatic representation called the Pascal Triangle, see Figure 4(a). Blaise Pascal (1623 - 1662 A.D.), a great French mathematician, scientist and philosopher, used it to solve problems related to chance in gambling. This would later become the foundation for Probability theory. (Also within Chaos theory.) There was already a Chinese version of this concept, see Figure 4(b), on the cover of Ssu Yuan Yu Chien; a work published in 1303 A.D. by Chu Shih-Chieh. (Peitgen 1992 [13]) This mathematical, geometrical object is actually a composite of the binomial coefficients of the polynomial $(1+x)^{n}$ which in terms of mathematics, represents an infinite resolution of the Sierpinski Gasket and Carpet, a typical icon of Chaos theory.

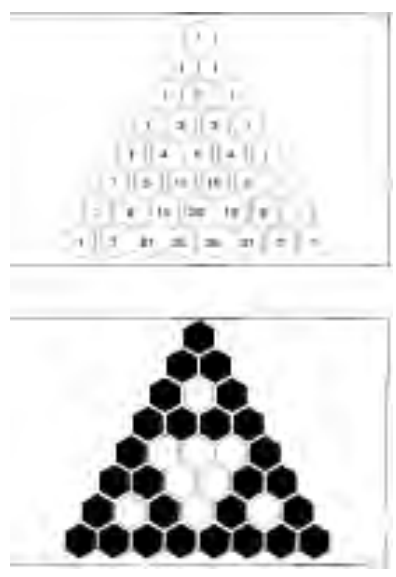

(a)

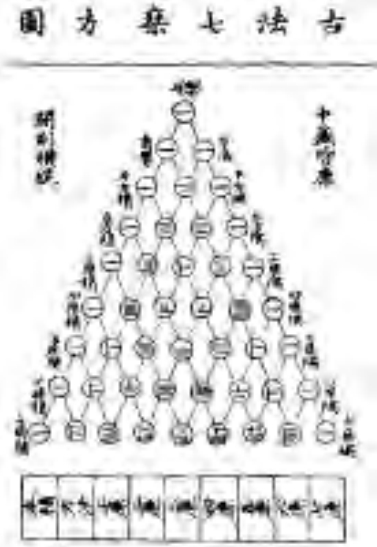

(b)
Figure 4: Pascal's triangle and Chinese manuscript from 1301 A.D. (From Peitgen, 1992 [13]).

From such descriptions of chaos theory by scientists, we can see a direct correlation with the description of Tao in the Tao Te Ching: "The 'power' that is most sufficing looks inadequate, ... stands firmest looks flimsy ... in its natural, pure state looks faded." (Lao Zi cited in Waley 1997 [18].)

Julia, Barnsley and Mandelbrot: these mathematicians give us new rules by which to generate geometrical shapes: that which are now known as Fractal Geometry. Unlike Euclidean and Cartesian methods - which use equations statically and turn them into curves, ellipses, parabolas, etc these new fractal geometricians use an equation as an actual process in itself rather than merely descriptive; as a dynamic process instead of merely static. The numbers applied to the equation are done so in order to produce a certain kind of behavior. "One behavior might be a steady state. Another might be a convergence to a periodic repetition of states. Another might be an out-of-control race to infinity." (Gleick 1987 [6].) Though the point generated by these processes behaves randomly, the final outcome always results in perfectly deterministic shapes.

The point being, that out of the chaotic, random approaches to the processes applied, we always end up with a global, universal outcome that is seemingly lawful yet infinite. This is what is now known as the so-called 'chaos game'. It demonstrates the interplay between randomness and deterministic fractals. A single set of rules somehow embodies the final shape and may result in much more complicated structures, yet the structure to which these rules are applied maintains its original character of configuration through scales which means self-similarity. Another way of describing such a bewitching method of shaping things is provided for us in Lao Zi's Tao Te Ching: "Tao never does, yet through it all things are done." Or, to put it another way: "Tao makes things what they are, but is not 
itself a thing" (cited in Sze 1959 [17]). A final and somewhat paradoxical description notes that: "They are called shapeless shapes; form without form; vague semblances." (Lao Zi cited in Waley 1997 [18].)
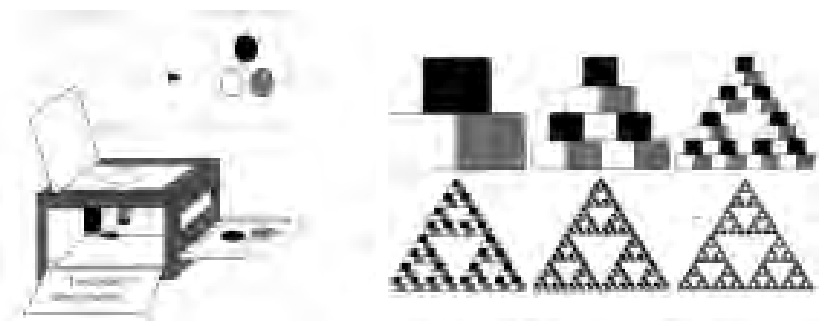

Figure 5: Multiple reduction machine and the Sierpinski gadget (from Peitgen, 1992 [13]).

Using so-called MRCM (multiple reduction copy machine) as a metaphor for what is known as deterministic iterated function system (IFS) in mathematics (see Figure 5 ), scientists can obtain a variety of fractal geometries, now regarded as a new language all of its own in mathematics.

"As the English language can be broken down into letters and the Chinese language into characters; fractal geometry promises to provide a means to break down the patterns and forms of nature into primitive elements, which can then be composed into 'words' and 'sentences' describing these forms efficiently." (Barnsley 1988 [1].)

"The evolution of Western science along its spiral path; starting from the mystical philosophies of the early Greeks, then rising and unfolding in an impressive development of intellectual thought. Increasingly turned away from its mystical origins to develop a world-view that is in sharp contrast to that of the Far East. In its most recent stages, Western Science is finally overcoming this view and coming back to those of the early Greek and Eastern philosophies." (Capra 1975 [3].)

\section{Regularity of Irregularity - Traditional Chinese Art and Fractals}

The strong mathematical and metaphysical accent of Tao suggests that the ancient Chinese sages and scholars were drawing upon the principle of paradox: a regularity of irregularity; a unity in diversity. Inevitably, parallels could also be found in the traditional art's technical principles and aesthetic evaluations.

\subsection{Subject Matter: Fractal Forms}

The first parallel emerges from the question as to why Chinese art is dominated by a profusion of mountains, rocks, trees, bamboo, weeds, clouds and other forms of nature. These diverse subject matters of Chinese art, which seem so distinct from each other in form, surprisingly belong to the same catalogue of fractal forms, if looked at from the standpoint of chaos theory. Such manifold forms are representative of various manifestations of the one universal principle or ultimate reality - Tao. Sze [17] asserts that in depicting these forms and their relationships to one another, the artist uses a symbolic language to reveal the underlying presence of Tao. In being a symbolic medium, art provides for a more direct route to the source than language otherwise could, with language being a far more abstracted medium. So painting provides a more vivid way of describing Tao. Painting is a carrier of the vital forces (chi) of Tao, providing a means in which to enlighten people, to heighten or awaken their senses and reconcile them with the ubiquitous Tao, giving the viewer a sense of this peace.

The chi notion in painting can perhaps, be better understood by presenting it as an analog to 'dusts' in the phase world of mathematics, whereby each 'dust' represents a dynamic system at a certain instant. It is an infinitesimal building block and carrier of vital force in Chinese philosophy and paintings. It is abstract scalar without physical substance, just like the 'dust' in phase space. When the chi in painting or the 'dusts' in phase imaging condense they both merge into dots and lines from which fractal shapes and forms emerge. Although chi itself has no form, through its movements and accumulations, all forms derive from it.

\subsection{One Stroke Theory: Dynamical Scaling Pro- cess and Self-similarity}

Shi Tao (1641-1717 A.D.), a master of painting in Chinese history, wrote a profound essay on art entitled "Sayings of Friar Bitter-Melon". In this essay, he introduces the notion of the one-stroke method:

"In the primeval past there was no method. The primeval chaos was not differentiated. When the primeval chaos was differentiated, method (law) was born. It was born of one-stroke. This one-stroke is that out of which all phenomena are born, applied by gods and to be applied by man. The establishment of this one-stroke method creates a method out of no-method, and a method, which covers all methods ... Just as one begins on a distant journey with a first step, so this one-stroke contains in itself the entire universe and beyond; with a myriad of possible strokes and ink all beginning and ending here, merely waiting to be seized upon. So the universe can be captured in a single stroke when the artists idea is clearly expressed, the execution well done." (Cited in Lin 1967 [9].)

When discussing the ink flow effects, he says:

"Where the brush and ink blend, cloudy forms are produced. Undifferentiated, such cloudy forms represent chaos, and to bring definition out of chaos, there is inevitably the single-stroke." (Cited Lin 1967 [9].)

There has always been debate in Chinese art circles about Shi Tao's "one-stroke method" theory. What did he 
mean by "one-stroke" here? Our understanding is that Shi Tao treats the process of painting as a complex, dynamical system, where each single stroke is considered as essential to the final outcome. Shi Tao's perspective is of Tao, which sees a natural form as in continuous flow and change. From Chaos theory's point of view, it makes perfect sense that a painting is actually made of multiple self-similar strokes; that the basic shape of each single stroke defines the final outcome of the forms. Modern fractal artists also have a similar feeling about artistic methods, as articulated by John Briggs, when speaking about fractal art:

"When painters juxtapose multiple self-similar forms and colors on canvas, or composers transform a sequence of notes into multiple self-similar forms by varying the rhythm and projecting the sequence of notes into different sections of the orchestra, they create a tension that gives birth to lucid ambiguities. Such artistic juxtapositions might be called "reflectaphors" because the self-similar forms reflect each other yet contains, like metaphors, a tension composed of similarities and differences between the terms. This reflectaphoric tension is so dynamic that it jars the brain into wonder, awe, perplexity, and a sense of unexpected truth or beauty." (Briggs 1992 [2].)

The reason why artistic strokes are self-similar is that the subject matters - natural forms - exhibit self-similar properties. As Guo Xi (1020-1090 A.D.), an artist and art critic from the Song Dynasty, describes a mountain in 'A Father's Instructions':

"A mountain looks this way close by, another way a few miles away, and yet another way from a distance of a dozen miles. Its shape changes at every step, the more the farther one goes. It looks this way from the front, another way from the side, and yet another way from the back. Its aspect changes from every angle, as many times as the point of view. Thus one must realize that a mountain contains in itself the shape of several dozen or a hundred mountains." (Lin 1967 [9].)

He describes here the self-similar property of mountains derived from his scrupulous observation. Armed with a scientific-like perspective, we can see not only the profiles and surface of mountains, but also their inner structures thanks to the new paradigm of fractal clusters. Such a range of perspectives suggests that objects like a mountain - are self-similar or more precisely self-affine when measured from within mathematical terms of Chaos theory. According to Gleick[6] the notion of self-similarity means symmetry across scale, of pattern inside of pattern. This principle pervades all fractals in Nature.

\subsection{Essence (li) and Spirit (Shen): The Fractal Dimension.}

This reflects one of the principles of Chinese painting: That visual resemblance has no importance in judging a painting's quality. What is important is to "capture the real essence (its $l i)$ " and "capture both the form and the spirit (its Shen)" (Jing Hao (A.D. 920) cited in Lin 1967 [9].) Throughout the literature of Chinese art, one may often encounter sentences like "penetrates all inner nature of things" or "regard to the inner law (li) of things." As Yen Kuang-lu (384-456 A.D.) notes:

"The purposes of drawings are three: one, to picture $l i$ (the inner law of things), as in the hexagrams; two, to represent ideas, as in writing; and three, to draw forms, as in drawings." (Lin 1967 [9].)

The cold and dry classic three-dimensional factor seems so irrelevant and improper here, in measuring seemingly erratic and chaotic forms in all scale levels. That might be the reason why ancient Chinese artists didn't consider this factor to be as important as it is considered in Western art. This may be the reason why Chinese painting doesn't have much emphasis on three-dimensional perspective scheme. It seems that they were mainly concerned with the invariable property of irregular forms, which they called $l i$ or shen. If an artist's insights and apperception are accurate in capturing this property, his picture will appeal to the viewers of his work, due to the Tao being successfully captured, distilled and conveyed to the audience, and will be considered to be good art in the Chinese tradition.

While Chinese artists sought to perfect $l i$ and shen intuitively, the computer confirmed that invariable parameters do exist within the irregular forms of nature. The measurement, referred to as the Fractal Dimension, is a numeric measurement which is used to describe patterns found in Nature and its forms; like seacoasts, river - networks, trees and galaxies.

"The fundamental idea is to assume that the two quantities - length, or surface, or volume on the one hand, and scale on the other - do not vary arbitrarily but rather are related by a law, a law which allows us to compute one quantity from the other. Given a self-similar structure, there is a relation between the reduction factor $s$ and the number of pieces $a$ into which the structure can be divided; and that is $a=\frac{1}{s^{d}}$ or equivalently $D=\log \frac{a}{\log 1 / s}$. $D$ is called the self-similarity dimension." (Peitgen 1992 [13].)

Russ[15] concludes the point for us where he notes: "Finding that human vision can apparently respond to fractal dimensions is further indirect evidence that this is an important physical property, that helps to describe the natural world." Correspondingly, Chinese painting often represents forms with essential brush strokes, encoding and symbolizing them through reduction according to artists' own visual compression.

\subsection{Contour Lines (gou-le) and Texture Lines (cun): Boundary Lines and Brightness Pat- terns}

One characteristic of Chinese painting is the exaggeration of outlines, see Lin [9]. They are used to represent 
almost everything that exists in the natural world. Traditional Chinese painting focuses upon both contour lines (gou-le) and texture lines (cun). For chaos theory scientists, Chinese artists are actually depicting in their works boundary lines, profiles of fractals and their differing degrees of brightness patterns. In studying these boundaries and patterns, and by transferring them into phase log-log plots (e.g. the Mandelbrot set), scientists discovered the relationship between the outwardly noisy appearances of various objects in nature with their underlying inner fractal properties. They were able to establish a system of values in which to gauge fractal dimensions; for example, the Hausdorf dimension; the Minkowske dimension; the Kolmogorov (box-counting) dimension; the Hurst coefficient and the Fourier analysis, as given in Russ [15]. By selecting different thresholds of brightness levels, the derived digital images can reveal the characteristic/s of local textures.

Lines became the primary suggestive characters in painting, as well as in calligraphy. Suggestiveness, not articulation, is the ideal in all Chinese art. Like a good lyrical poem, "the number of words is limited, but the ideas it suggests are limitless." (Fung 1991 [5].) Perhaps Zong Bing (375 - 443 A.D.) articulates the point best for us where he notes:

The aesthetic evaluation of these instrumental lines is a subject of vigorous discourse in Chinese art history and literature. Brushstroke lines represent the principal technical instrumentality within Chinese painting. Even Chinese written language - is prefaced upon this symbolically preferred system. As Sze [17] states, in writing a Chinese character, one requires a finely tuned sense of proportion along with a subtle discrimination. This sense of fitness of form called "dynamic asymmetry", is prized by Chinese artists and scholars, and is a criterion of judgment. "A calligrapher conceived of each character as set into a nine-unit square; this integrated the character's disparate elements into a harmonious whole. Setting a character into a halved or quartered square destroyed its balance." (Sze [17]). See Figure 6 for an illustration of this effect.

Such a methodology is strikingly similar to the Kolmogorov or box-counting dimension, "which is determined by placing grids with different resolutions over a fractal profile and plotting (on $\log -\log$ axes) the number of grid squares through which the profile passes as a function of grid size." (Russ [15].) Could the sense of "dynamic asymmetry" of ancient Chinese art theorists equate with the fractal dimension, balance and harmony of the character?

This nine-grid unit is the so-called Nine Palaces, see Figure 7, in Taoism, a variation of a ba-gua (eight trigrams) diagram used to describe changes as given in Wong
[19]. And it is used to measure forms in a recursive manner, which means by various scale levels. The numbers in Figure 7 illustrate the "root structure" of the Nine Palaces. Such an arrangement of numbers in a grid does not seem strange to scientists of Chaos theory. By making constructions of classical fractals such as the Sierpinski Carpet, an identical arrangement of transforming the unit square into nine congruent sub-squares is used. Chaos theory scientists refer to such a configuration as a triadic coordinate system, see Peitgen [13].

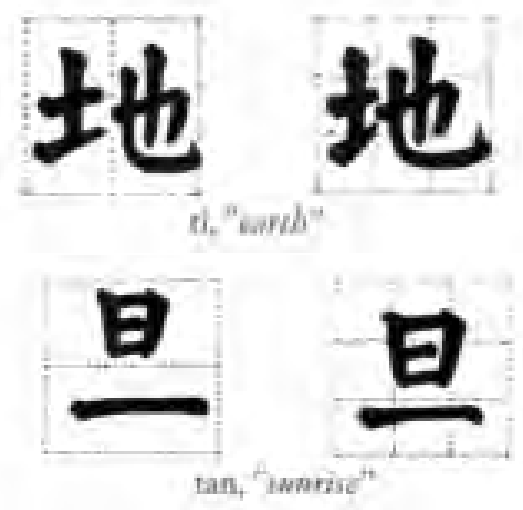

Figure 6: The well developed character, from Fitzgerald [4].

\begin{tabular}{|l|l|l|}
\hline 4 & 9 & 2 \\
\hline 3 & 5 & 7 \\
\hline 8 & 1 & 6 \\
\hline
\end{tabular}

Figure 7: The root structure of the Nine Palaces (Wong [19]).

The aesthetic judgment of calligraphy relies on an intrinsic mathematical property congenial to notions in Chaos theory. We might now understand why Chinese paintings are often juxtaposed with seal prints and calligraphic scripts. Such stamps and scripts were often poems, letters or simply diaries of the author. Besides the appreciation of the literary value of the scripts, the calligraphy itself, and the chops, belong to the same visual aesthetic category. In this context, Chinese painting combines text and image; both judged (valued) from a similar criteria.

The discussion above exemplified several Chinese art principles, from the perspective of Chaos theory's fractal notions. Such studies answered some of our questions about Chinese art. We wondered how a branch or a cluster of branches crossing a pictorial space could be decorated or represented by a series of dots and dashes, which of themselves bore no physical semblance to the form, yet would provoke a sense of these natural forms? How a seemingly 
arbitrary and chaotic rendering of sparse brushstrokes in a traditional Chinese painting could somehow resolve into a harmonious and ordered totality, successfully suggesting natural forms? How does the visual recognition become established? Chaos theory suggests that it is the intricate fractal aspect of Nature; asymmetrical and erratic as it is, that reveals these underlying forces that govern energies into spontaneous patterns of forms. Traditional Chinese art principles and its accumulation of practical experience also rest upon this fuzzy and dusty foundation of criticality - of a regularity of irregularity, of a unity in diversity.

\section{Discussion and Analysis of Brushworks - Symbols of Art \\ 4.1 The Tension of Forms}

The significance of Chinese painting lies within its abstractive tendency. Just as the western impressionist movement found an aesthetic tension among different colors, thereby discovering the atlas of the color spectrum system, Chinese art revealed and discovered that there is also another existing atlas of forms - a spectrum of complexity.

The seemingly erratic, random and discrete appearances of forms in nature are revealed to be phenomena that exhibit an underlying consistency. There is a uniformity of degrees in the irregularities of forms and shapes in Nature. Such a degree can be measured by chaos theory in the category of fractals. The so-called $\log -\log$ plot of this theory provides us with a value that scientists name the fractal dimension. This seemingly artificial intellectual value exists intrinsically within every perceivable structure in nature. They even exist within the very marks and tracks we make to represent them through our behavior and activities in making any image.

Human vision has been shown to respond to this fractal dimensional value instinctively, as naturally as we respond to colors and sounds. "Finding that human vision can apparently respond to fractal dimension is a further indirect evidence that this is an important physical property that helps to describe the natural world." (Russ 1994 [15].) Our senses define a color by the intrinsic vibration value of its light-wave frequency. Radio-waves are detected in a similar fashion, by frequency. From a scientific perspective, color and sound are just two phenomena measurable within the same category of electromagnetic waves. The only difference between them is the frequency. So what about forms and shapes?

The universe is an interactive and intertwined mixture of forces and phenomena. Order only exists in a "selforganized criticality." In Chinese art theory, it is considered as the spontaneity (zi-ran) and truth (zhen) of Nature, which artists should be aware of and seek to catch up with, as order is also ever changing. The evaluation of art may well rest upon this intriguing aspect of Nature in which or- der and disorder coexist at the same time. If this is the case, then artists should be alert to and sensitive about the intermingled nature of the ever changing and conflicting factors and forces that shape our world.

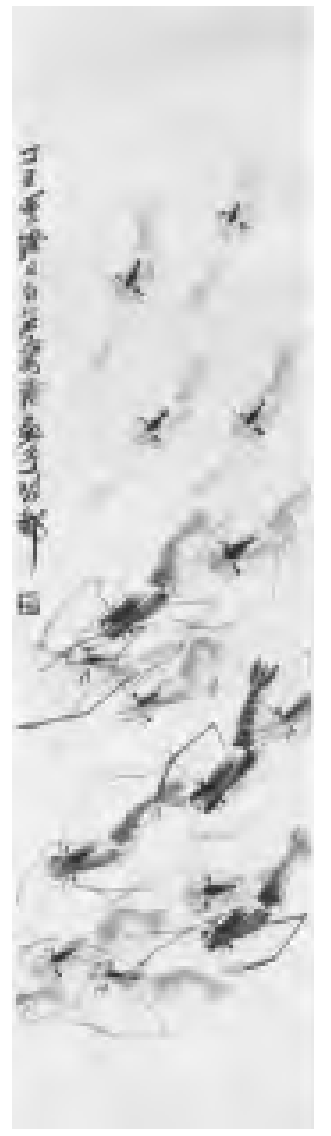

Figure 8: Chi Bo-shi's (1863-1957 A.D.) work of Yabbies.

This tension, inherent within the subtle order in nature, exists everywhere and in all things. Anything: a blade of grass or an orchid; a rose bud or a lotus flower; a bamboo shoot or a plum tree; can exhibit this tendency when juxtaposed within a single work of art. Even a panoramic landscape in which relationships become more grand and sophisticated, involving seasonal changes and incorporating geographical factors as they do, adhere to such a tension. There exists then, an endless possibility of weaving forms together. Take Chi Bo-shi's (1863-1957 A.D.) work of Yabbies, see Figure 8, for example. Before him, nobody had ever tried to pick this form as a subject matter, yet he successfully invented the unique sets of strokes and methods that are now utilized in order to depict the form. By composing these Yabbies into a group, just as they move and appear in nature, he created a rhythm and tension amongst the forms in which they appear to be alive in the water, though there is not a single delineation of cur- 
rents in the picture to signify any presence or movement of water. The relationship between the yabbies and water is implicitly present in the simplest and most concise way. Such a terse treatment of brush-works reaches its highest standard of aesthetic achievement in Chinese artworks.

\section{Fractal Dimensional Value of Brush-work}

The aim of building up a picture is to establish a network of tension and order among brush-strokes. This tension and order ultimately depends upon the personified and codified symbols and marks set down by the artist.

"The brush-stroke is the backbone of a painting. For trees and rocks have no regular fixed shapes, but are fixed by the brush. The shapes and forms change infinitely as the brush touches the paper at the artist's will." (Shen Zongji, cited in Lin [9].)

The principle expressive medium of Chinese painting is achieved through instrumental lines. In fact, the contour lines (goule) and texture lines (cun) are made up of dots, dashes and strokes. "Each brushstroke should be a living idea. Brushwork is thus the direct expression of the mind in action. Its function is to make visible the invisible." (Sze [17].) How then do these rhythmic dots, dashes and lines make sense to our vision in creating an aesthetic tension? And what exactly is the invisible?

These dots, dashes and strokes, are nothing other than fractal shapes. There is no such thing as regularity in the shapes of brushstrokes in art, just as there are no regular, identical shapes in Nature. Traditional Chinese artists often speak of brush strokes metaphorically, associating with forms in Nature - the dot that looks like a "falling stone", a "tiger's claw", an "eagle's beak", etc.; Or the stroke which resembles a "nail-head", a "rat's tail", an "axe-cut", etc. (from Kwo [8].) So artists should be very careful in constructing and arranging these marks in an attempt to represent a form from Nature.

In the following we analyze the brush-works in the Mustard Seed Garden Manual of Painting[12] and some works by ancient Chinese artists, to determine the fractal dimensions of common (Bamboo, Plum tree, weeds, and rocks) elements in traditional Chinese Art.

\subsection{Bamboo}

Here we consider four traditional Chinese artists renditions of Bamboo. These are Wen Tong (Song dynasty), see Figure 9, Guan Daoshen (1262-1319 A.D.), and Lin Yang (1245-1320 A.D.), and bamboo in a composite image by Xi Fangya (Yuan dynasty).

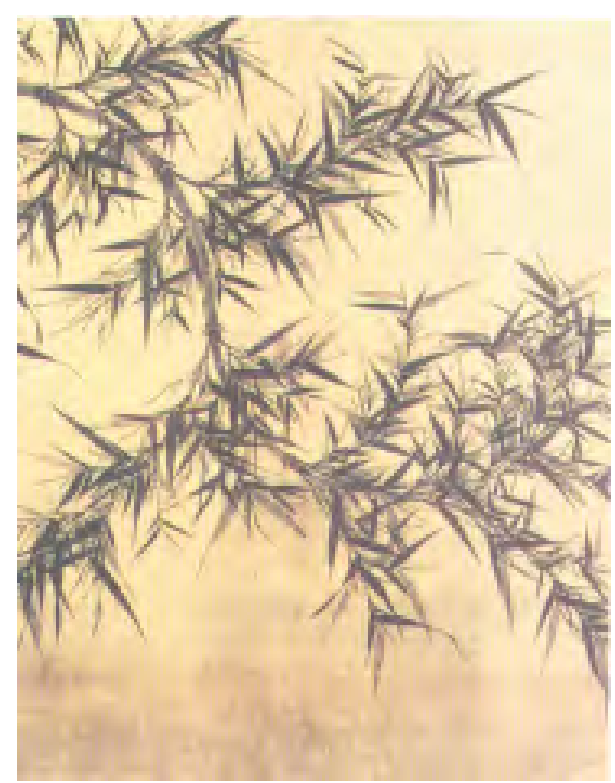

Figure 9: Wen Tong, Song dynasty. Ink on silk, $131.6 \mathrm{~cm}$ long and $105.4 \mathrm{~cm}$ high.

The box-counting dimension of the work by Wen Tong yields a fractal dimensional value of between $1.80-1.84$. The artist Li Yan's work consists of two kinds of bamboo and the box-counting dimension of the work yields a fractal dimensional value of between 1.84-1.85. The artist Guan Daoshen work when analysed using the box counting method yields a fractal dimensional value of around 1.77 - 1.80. Finally the artist Xi Fangya work yields a fractal dimensional values of between 1.73-1.86 for the various bamboo images.

These bamboo works are depicted in different styles and are portrayed in a range of compositions over a long period of time, yet each individual form of bamboo conforms to a fractal dimensional value of between $1.73-1.86$. It would appear that these Chinese artists somehow understood the intrinsic properties of bamboo forms. What parameter does real bamboo yield? By examining some local bamboo, and exposing it to a similar analysis of its fractal dimensional value, we discovered that it yielded a value of approximately 1.78 , which falls within the same data range of the bamboo depicted in traditional Chinese painting.

\subsection{Plum}

Figure 10 shows Plum images from a painting from the work of the artist Yang Wujiu (1097 - 1169 A.D.). 


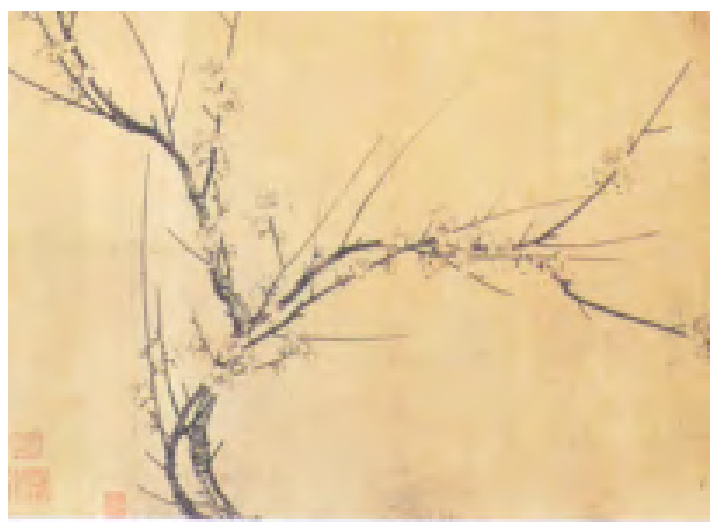

Figure 10: Plum from Yang Wujiu (1097 - 1169 A.D.) Ink on paper, $37.2 \mathrm{~cm}$ high, $358.8 \mathrm{~cm}$ long.

The box-counting fractal dimension value for the Plum trees in this and other work we analysed is between 1.58 and 1.67 .

\subsection{Weeds}

The paintings of weeds, in Figure 11, are taken from “The Mustard Seed Garden Manual of Painting"[12]. The corresponding fractal dimension values vary from 1.51 to 1.74 for these paintings.

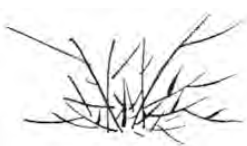

(a)

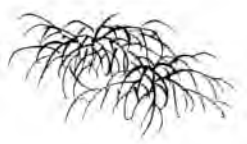

(c)

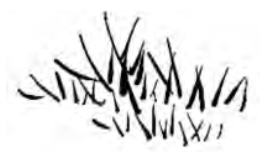

(b)

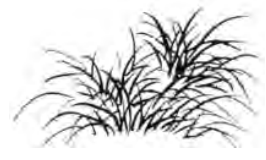

(d)
Figure 11: Pictures of weeds from the Mustard Seed Garden Manual of Painting[12].

\subsection{Rocks}

The following paintings of rocks, in Figure 12, are taken from "The Mustard Seed Garden Manual of Painting" [12].

The fractal dimensions for both the outlines of the rocks and the interior shading where calculated. The fractal dimension values vary from 1.32 to 1.42 for the outlines and from 1.60 to 1.75 for the interiors of these paintings.

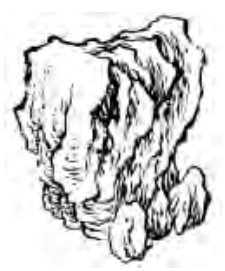

(a)

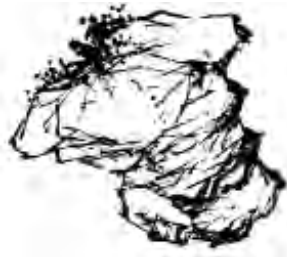

(c)

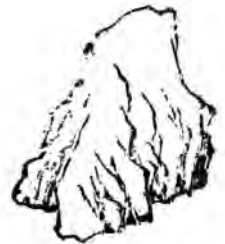

(b)

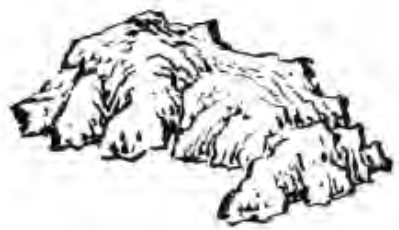

(d)
Figure 12: Pictures of rocks from the Mustard Seed Garden Manual of Painting[12].

\subsection{Discussion}

Several interesting facts are revealed by these studies. Firstly, that the fractal dimensional value serves as a decisive parameter for Chinese artists to conform to in order for their work to be accepted as an accurate rendering. We can see from the individual artists' works above, their acute awareness of the differences among various wild branches and structures in Nature along with their ability to distinguish between them in drawing and painting. To these ancient Taoist artists, the seemingly untamable and chaotic world of wild patterns in Nature becomes a delightful guide for their simplified and abstract brush works.

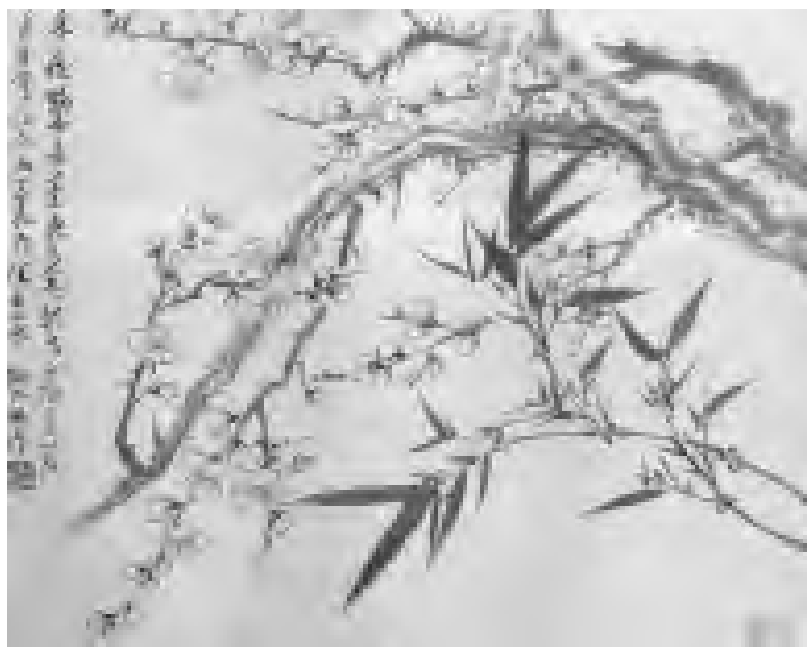

Figure 13: Bamboo and Plums from Yi-fei Lu[10].

With fractal values in mind, it becomes understandable why an artist uses a bamboo branch against a plum in order to compliment and yet distinguish between the two forms. This comparative and contrasting work serves to bring to consciousness such a tension. The choice of the two forms 
is not arbitrary but serves to yield a definitive effect in capturing and representing the essential differences present in each form. The reason this works as an artistic creation is due to the varying yet complimentary fractal values $(1.67$ vs. 1.72) between the two forms, as seen in Figure 13. Our vision then, somehow responds instinctively to this complimentary difference, like two different colors or two musical tones. The forms create a tension or a chord in the minds' eye that synthesizes into a harmony between the two lively plants. However, if each form were not properly delineated, according to its intrinsic value or in Chinese phraseology, its "virtue" ( $d e)$, then the result and effect would be marred. Creating a similar effect to a confusing chord in music.

This pairing of forms could be extended into a trio or a quartet by adding other fractal forms such as rocks and weeds. Even more delicate forms such as insects and birds can also be incorporated into a work depending upon seasonal factors and ecological propriety. The possibilities are endless. The art form reaches its full realization and climaxes in panoramic mountain-scapes and landscapes - in a sheer visual symphony. The end result causes the tension and harmony of fractal forms to be more subtle and exquisite.

Strokes of similar objects appear proportional. As highlighted above, in measuring the strokes that are used in depicting rocks, the fractal dimension values of the outline and the texture lines of a rock are mutually complimentary. They support each other by giving a total combined fractal dimensional value of almost 3 . It seems that the two aspects of rock lines, when measured together give a three-dimensional sense of the object, both intrinsically and mathematically, provided they are set up correctly and proportionally.

The significance of the Traditional Chinese art form lies in capturing essences of forms, rather than an exact rendering. In chaos theory, it equates to creating different fractal dimensional values of fractal forms. Such forms inform the human vision on a purely instinctive and intuitive level. In allowing the eye to wander and meander over the picture, one is confronted with a multiplicity of similar strokes that dissolve into different fractal structures. The overall effect is in capturing a sense of the form the artist is depicting, so that one's previous experiences of a similar image is triggered, and a recognition of the subject matter is garnered.

Chinese traditional art forms are depicted on long scrolls both vertically and horizontally, in an attempt at displaying a dynamic viewing medium. From a chaos theory perspective, the viewer is actually subconsciously calculating and accumulating various fractal dimensional values in a dynamic way until they become aware of the forms reflected from previous visual experiences in the real world or the world of art. Yet these artificial and instrumental strokes remain intrusively abstract and personified, so accidental ambiguities remain. The process of appreciation extends to the viewers' personal and spiritual preferences and imagination that go far beyond the pictorial representation. Hence the artists' aesthetic enlightenment can actually be measured and conveyed.

Simplicity and economy of visual interpretation is essential in this limited medium of art. A myriad of natural forms can be generated using the same simple, yet paradoxical format. The numeric nuances that underlie natural forms constitute the pattern of recognition in our sensory system, which enables this dynamic and flexible approach to visual interpretation. This flexibility is also present in the style of, and through the interpretation of, each individual artist. Although intuitive in their rendering, it is the underlying mathematic truth of fractal dimensional values that gives each symbol its suggestive power.

We explored the use of fractal forms in developing a traditional Chinese aesthetic artwork where the use of fractals is made to animate the individual brushstrokes. In this way the Plum tree can be made to come to life as the tree grows and buds, and the bamboo leaves move in the wind. The Yabbie and Goldfish forms can come alive and seem to move in a 3D space even though the brushstrokes are all 2D. A Chinese CG artwork giving expression to these ideas can be found at http://www.youtube.com/watch?v=LKFKxfL7tV4. This work won a gold award at ACMSiggraph ComGraph 2006 in Singapore, and $1^{\text {st }}$ prize at the Art of Digital Show, Oct. 6-Nov 19, 2006 at San Diego.

Some individual frames from the animated Chinese CG is given in Figure 20(a)-20(d). An individual brushstroke becomes the underlying fractal atom by which forms are created. The fractal brushstrokes are formed along a 3D spline in Maya. Thus the brushstrokes can be transformed in 3D space over time to create an animation. Multiple instances of the forms can be used to add complexity to the scene. This usage allows us to create a modern animated Chinese art form that reflects the traditions of the classical Chinese style.

In the future we intend to extend this work to further classify Chinese art forms based on the fractal dimensions of the underlying form. We also intend to explore Chinese calligraphy to determine what, if any, fractal or chaotic behavior can be determined for these forms.

In this work we have have linked the notions of the Tao, the principles of Chinese Art and Calligraphy, to the notions found in fractal and chaos theory. We have explored how these notions result in similar philosophical and aesthetic ideals. Utilizing these notions we have developed a modern version of animated brushwork, where the individ- 
ual brushstrokes (fractal self similar images) come alive. This establishing a link between these notions that span both time and culture.

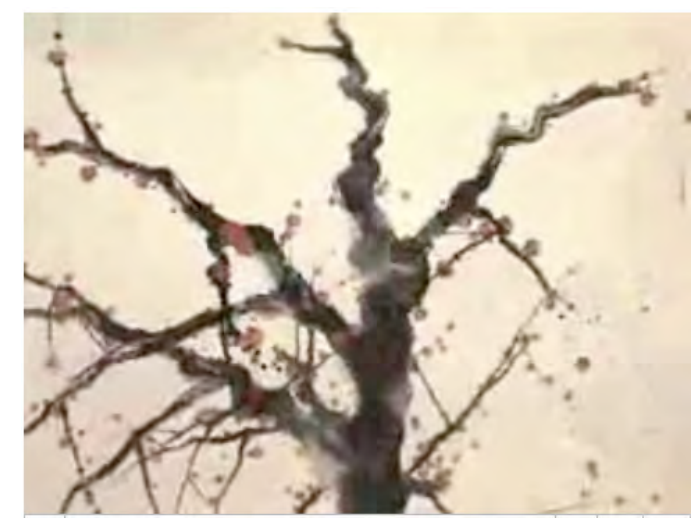

(a)

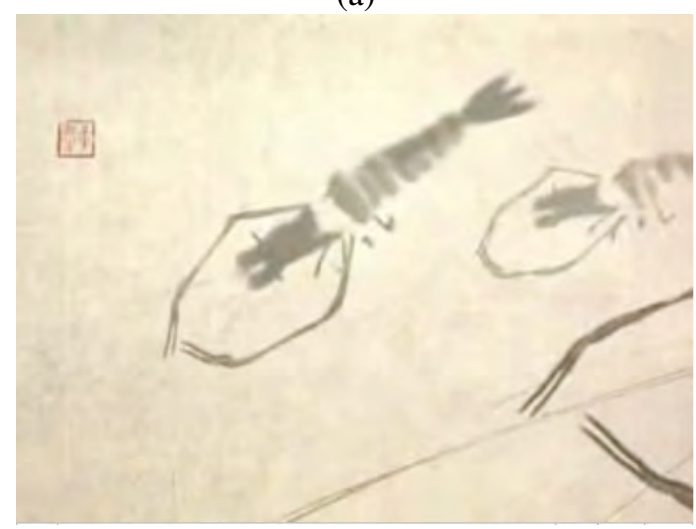

(b)

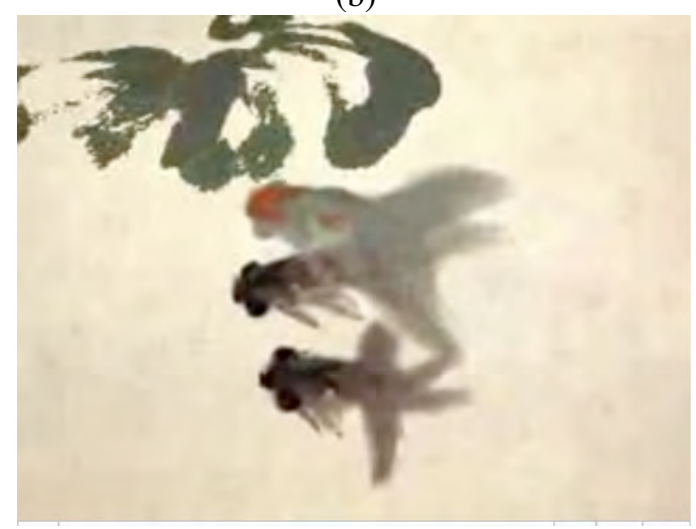

(c)

Figure 14: Single frames from Chinese CG artwork at http://www.youtube.com/watch?v=LKFKxfL7tV4, showing animated (a) Plum tree (b) Yabbies, and (c) Goldfish. The animation uses fractal strokes to animate these objects.

\section{Acknowledgement}

We would like to acknowledge support for this project from our University.

\section{References}

[1] Barnsley, M. F., Devaney, R. L., Mandelbrot, B. B. and Voss, R. F., 1988. The Science of Fractal Images. New York: Springer-Verlag New York Inc.

[2] Briggs, J., 1992. Fractals: The Patterns of Chaos. London: Thames and Hudson Ltd.

[3] Capra, F., 1975. The Tao of Physics. Boston: Shambhala Publications, Inc.

[4] Fitzgerald, C. P., 1969. The horizon history of China. New York: American Heritage Publishing Co., Inc.

[5] Fung, Y. L., 1991. Selected Philosophical Writings of Fung Yu-lan. Beijing: Foreign Languages Press.

[6] Gleick, J., 1987. Chaos. London: Sphere Books Ltd.

[7] Greenfield S., 2000. Brain Story. London: BBC Worldwide Ltd.

[8] Kwo, Da-wei., 1981. Chinese Brushwork: Its History, Aesthetics, and Techniques. Totowa: Allanheld, Osmun \& Co.

[9] Lin, Y. T., 1967. The Chinese Theory of Art. London: William Heinemann Ltd.

[10] Lu, Yi-fei., 1997. Collections of Rongbao House Shanghai: Rongbao House Publishers.

[11] Mandelbrot, B. B., 1977. The Fractal Geometry of Nature. New York: W. H. Freeman and company.

[12] 1982. Mustard Seed Garden Manual of Painting Shanghai. Shanghai Bookstore Publishers.

[13] Peitgen, H. O., Jrgens, H. and Saupe, D., 1992. Chaos and Fractals: New Frontiers of Science. SpringerVerlag New York, Inc.

[14] Ruelle D., 1985. Chance and Chaos. London: Penguin Books Ltd.

[15] Russ, C. J., 1994. Fractal Surfaces. New York: A Division of Plenum Publishing Corporation.

[16] Smith, D. L., 1980. The wisdom of Taoist mystics. London: Sheldon Press.

[17] Sze, M. M., 1959. The Way of Chinese Painting. New York: Random House, Inc.

[18] Waley, A., 1997. Tao Te Ching. Hertfordshire: Wordsworth Editions Ltd.

[19] Wong E., 1997. The Shambhala Guide to Taoism. Boston and London: Shambhala Publications, Inc. 